\title{
Higgs production through gluon fusion: Updated cross sections at the Tevatron and the LHC
}

\author{
Daniel de Florian ${ }^{\mathrm{a}, \mathrm{b}, *}$, Massimiliano Grazzini ${ }^{\mathrm{c}}$ \\ a Departamento de Física, FCEYN, Universidad de Buenos Aires, (1428) Pabellón 1, Ciudad Universitaria, Capital Federal, Argentina \\ b Physics Department, Brookhaven National Laboratory, Upton, NY 11973, USA \\ c INFN, Sezione di Firenze and Dipartimento di Fisica, Università di Firenze, I-50019 Sesto Fiorentino, Florence, Italy
}

\section{A R T I C L E I N F O}

\section{Article history:}

Received 19 January 2009

Received in revised form 3 March 2009

Accepted 20 March 2009

Available online 26 March 2009

Editor: G.F. Giudice

\begin{abstract}
A B S T R A C T
We present updated predictions for the total cross section for Higgs boson production by gluongluon fusion in hadron collisions. Our calculation includes the most advanced theoretical information available at present for this observable: soft-gluon resummation up to next-to-next-to-leading logarithmic accuracy, the exact treatment of the bottom-quark contribution up to next-to-leading order, and twoloop electroweak effects. We adopt the most recent parametrization of parton distribution functions at next-to-next-to-leading order, and we evaluate the corresponding uncertainties. In comparison with our previous central predictions, at the Tevatron the difference ranges from $+9 \%$ for $m_{H}=115 \mathrm{GeV}$ to $-9 \%$ for $m_{H}=200 \mathrm{GeV}$. At the LHC the cross section is instead significantly increased. The effect goes from $+30 \%$ for $m_{H}=115 \mathrm{GeV}$ to $+9 \%$ for $m_{H}=300 \mathrm{GeV}$, and is mostly due to the new parton distribution functions. We also provide new predictions for the LHC at $\sqrt{s}=10 \mathrm{TeV}$.
\end{abstract}

(c) 2009 Elsevier B.V. All rights reserved.
The Higgs boson is a key ingredient of the Standard Model (SM), but it has so far eluded experimental discovery. Direct searches at LEP lead to a 95\% CL lower limit of $m_{H}>114.4 \mathrm{GeV}$ on the mass $m_{H}$ of the SM Higgs boson [1]. At the LHC the Higgs boson can be discovered over the full mass range up to $m_{H} \sim 1 \mathrm{TeV}$ within a few years of running. At the Tevatron, the CDF and D0 experiments are now becoming sensitive to a Higgs signal at $m_{H} \sim 170 \mathrm{GeV}[2]$.

The dominant mechanism for SM Higgs boson production at hadron colliders is gluon-gluon fusion, through a heavy-quark (mainly, top-quark) loop. When combined with the decay channels $H \rightarrow \gamma \gamma$ and $H \rightarrow Z Z$, this production mechanism is one of the most important for Higgs boson searches and studies over the entire range, $100 \mathrm{GeV} \lesssim m_{H} \lesssim 1 \mathrm{TeV}$, of Higgs boson mass to be investigated at the LHC. In the mass range $140 \mathrm{GeV} \lesssim m_{H} \lesssim 180 \mathrm{GeV}$, gluon fusion, followed by the decay $H \rightarrow W W \rightarrow l v l v$, offers the main discovery channel of the Higgs boson at the LHC and also at the Tevatron, thanks to the strong angular correlations of the charged leptons in the final state.

In QCD perturbation theory the leading order (LO) contribution to the $g g \rightarrow H$ cross section is proportional to $\alpha_{S}^{2}$, $\alpha_{\mathrm{S}}$ being the QCD coupling. The QCD corrections have been computed at nextto-leading order (NLO) $[3,4]$ in the heavy-top limit, and with full

\footnotetext{
* Corresponding author at: Departamento de Física, FCEYN, Universidad de Buenos Aires, (1428) Pabellón 1, Ciudad Universitaria, Capital Federal, Argentina.

E-mail addresses: deflo@df.uba.ar (D. de Florian), grazzini@fi.infn.it (M. Grazzini).
}

dependence on the masses of the top and bottom quarks [4]. Nextto-next-to-leading order (NNLO) corrections have been obtained in the heavy-top limit [5]. These QCD corrections, which are dominated by radiation of soft and virtual gluons [6], lead to a substantial increase of the LO result. The QCD computation up to NNLO has been consistently improved by adding the resummation of soft-gluon logarithmic contributions, up to next-to-next-to-leading logarithmic (NNLL) accuracy [7]. The ensuing NNLL + NNLO results are nicely confirmed by the more recent computation [8-10] of some of the soft-gluon terms at $\mathrm{N}^{3} \mathrm{LO}{ }^{1}$

In this Letter we present an update to the NNLL + NNLO results of Ref. [7]. We refrain from repeating the theoretical details of the calculation, which can be found in Ref. [7], and we only describe the main changes implemented in the present work. In our previous analysis the benchmark predictions were obtained using the MRST2002 NNLO partons [11]. Since then, there have been important modifications in the extraction of parton distribution functions (PDFs). The calculation of the NNLO splitting functions ${ }^{2}$ was completed [12]. Furthermore, a more sophisticated treatment of heavyquark thresholds has been introduced in [14], resulting in a significant modification of the gluon and light quark densities in the recent MSTW2008 set [15]. For example, at $x \sim 0.01$, relevant for

\footnotetext{
1 Note, however, that the perturbative information available at present does not allow to consistently extend the computation to $N^{3} \mathrm{LL}$ accuracy.

2 The MRST2002 fit was based on approximate expressions for the evolution kernels [13].
} 
a Higgs boson of $m_{H} \sim 120 \mathrm{GeV}$ at the LHC, the gluon distribution increases by about $6 \%$ with respect to the MRST2002 fit. The value of $\alpha_{\mathrm{S}}\left(m_{Z}\right)$ also had a non-negligible change from 0.1154 to 0.1171 . Considering that the total cross section is completely dominated by the gluon-gluon fusion channel, and that the lowest order contribution starts at $\mathcal{O}\left(\alpha_{\mathrm{S}}^{2}\right)$, with sizeable corrections at higher orders, it is not surprising that the mere change from MRST2002 to MSTW2008 partons can result in an increase of more than 10\% in the production cross section, making an update mandatory. The change in the PDFs does not result in such a dramatic increase of the cross section at the Tevatron, since at $x \sim 0.06$, relevant for the production of a Higgs boson of $m_{H} \sim 120 \mathrm{GeV}$, the gluon distribution is reduced by about $4 \%$, but the decrease is partially compensated by the increase of the partonic cross section due to the larger coupling constant.

Besides the important effect of the PDFs, there are other theoretical reasons for revisiting the computation of the Higgs cross section at hadron colliders. In particular there has been an important effort to evaluate the electroweak (EW) corrections arising from $W$ and $Z$ boson coupling to the Higgs and to both light and heavy quarks in the loop [16]. The recent computation of Ref. [17] takes into account those contributions by avoiding the complications in the two-particle threshold using the complexmass scheme. The EW corrections turn out to be of the order of a few percent, with a sign depending on the Higgs mass. The main uncertainty in the EW analysis comes from the fact that it is not completely clear how to take them into account in practice. In the partial factorization scheme of Ref. [17] the EW correction applies only to the LO result. In the complete factorization scheme instead, the EW correction multiplies the full QCD corrected cross section. Since QCD corrections are sizeable, the latter choice has a non-negligible effect on the actual impact of EW corrections in the computation. The recent analysis of higher-order QCD and EW corrections presented in Ref. [18], performed on the basis of an effective Lagrangian approach, supports the complete factorization hypothesis, suggesting that EW corrections become, to a good approximation, a multiplicative factor of the full QCD expansion.

The predictions we present below are obtained as follows. We first consider the top-quark contribution in the loop, and perform the calculation up NNLL + NNLO in the large- $m_{t}$ limit. The result is rescaled by the exact $m_{t}$ dependent Born cross section, since this is known to be an excellent approximation for the top-quark contribution. This resummed top-quark contribution provides the bulk of the Higgs cross section at hadron colliders. We then consider the bottom-quark contribution (more precisely, the bottom contribution and the top-bottom interference). Since in this case the effective theory approach is not applicable, we follow Ref. [18] and we include this contribution up to NLO only (but still computed with NNLO MSTW2008 partons), by using the program HIGLU [4]. Finally, we correct the result by including the EW effects evaluated in Ref. [17] in the complete factorization hypothesis. We set the heavy-quark masses to $m_{t}=170.9 \mathrm{GeV}$ and $m_{b}=4.75 \mathrm{GeV}$, the latter consistently with the MSTW2008 set. Our central predictions $\left(\sigma^{\text {best }}\right)$ are obtained by setting the factorization $\left(\mu_{F}\right)$ and renormalization $\left(\mu_{R}\right)$ scales equal to the Higgs boson mass.

Our results for the Tevatron at $\sqrt{s}=1.96 \mathrm{TeV}$ and the LHC at $\sqrt{s}=10 \mathrm{TeV}$ and $\sqrt{s}=14 \mathrm{TeV}$ are presented in Tables 1,2 and 3, respectively. Comparing to our previous predictions (see Tables 1 and 2 of Ref. [7]), the cross sections change significantly. At the Tevatron the effect ranges from $+9 \%$ for $m_{H}=115 \mathrm{GeV}$ to $-9 \%$ for $m_{H}=200 \mathrm{GeV}$. At the LHC the effect goes from $+30 \%$ for $m_{H}=$ $115 \mathrm{GeV}$ to $+9 \%$ for $m_{H}=300 \mathrm{GeV}$. It is worth noticing that at the LHC more than half of the increase arises from the modification in the gluon distribution and the coupling constant.

The bottom contribution, dominated by bottom-top interference, is small and negative. The different treatment of this con-
Table 1

Cross sections (in pb) at the Tevatron $\left(\mu_{F}=\mu_{R}=m_{H}\right)$ with $\sqrt{s}=1.96 \mathrm{TeV}$, using the MSTW2008 [15] parton densities.

\begin{tabular}{|c|c|c|c|}
\hline$m_{H}$ & $\sigma^{\text {best }}$ & Scale & PDF \\
\hline 100 & 1.861 & $\begin{array}{l}+0.192 \\
-0.174\end{array}$ & $\begin{array}{l}+0.094 \\
-0.101\end{array}$ \\
\hline 105 & 1.618 & $\begin{array}{l}+0.165 \\
-0.149\end{array}$ & $\begin{array}{l}+0.085 \\
-0.091\end{array}$ \\
\hline 110 & 1.413 & $\begin{array}{l}+0.142 \\
-0.127\end{array}$ & $\begin{array}{l}+0.077 \\
-0.083\end{array}$ \\
\hline 115 & 1.240 & $\begin{array}{l}+0.123 \\
{ }_{-0.110}\end{array}$ & $\begin{array}{l}+0.070 \\
-0.075\end{array}$ \\
\hline 120 & 1.093 & $\begin{array}{l}+0.107 \\
-0.095\end{array}$ & $\begin{array}{l}+0.065 \\
-0.069\end{array}$ \\
\hline 125 & 0.967 & $\begin{array}{l}+0.094 \\
-0.083\end{array}$ & $\begin{array}{l}+0.059 \\
-0.063\end{array}$ \\
\hline 130 & 0.858 & $\begin{array}{l}+0.082 \\
-0.072\end{array}$ & $\begin{array}{l}+0.054 \\
-0.058\end{array}$ \\
\hline 135 & 0.764 & $\begin{array}{l}+0.073 \\
-0.063\end{array}$ & $\begin{array}{l}+0.050 \\
-0.053\end{array}$ \\
\hline 140 & 0.682 & $\begin{array}{l}+0.065 \\
-0.056\end{array}$ & $\begin{array}{l}+0.046 \\
-0.049\end{array}$ \\
\hline 145 & 0.611 & $\begin{array}{l}+0.057 \\
-0.049\end{array}$ & $\begin{array}{l}+0.042 \\
-0.045\end{array}$ \\
\hline 150 & 0.548 & $\begin{array}{l}+0.051 \\
-0.044\end{array}$ & $\begin{array}{l}+0.039 \\
-0.042\end{array}$ \\
\hline 155 & 0.492 & $\begin{array}{l}+0.045 \\
-0.039\end{array}$ & $\begin{array}{l}+0.036 \\
-0.038\end{array}$ \\
\hline 160 & 0.439 & $\begin{array}{l}+0.040 \\
-0.034\end{array}$ & $\begin{array}{l}+0.033 \\
-0.035\end{array}$ \\
\hline 165 & 0.389 & $\begin{array}{l}+0.036 \\
-0.030\end{array}$ & $\begin{array}{l}+0.030 \\
-0.032\end{array}$ \\
\hline 170 & 0.349 & $\begin{array}{l}+0.032 \\
-0.027\end{array}$ & $\begin{array}{l}+0.028 \\
-0.029\end{array}$ \\
\hline 175 & 0.314 & $\begin{array}{l}+0.029 \\
-0.024\end{array}$ & $\begin{array}{l}+0.026 \\
-0.027\end{array}$ \\
\hline 180 & 0.283 & $\begin{array}{l}+0.026 \\
{ }_{-0.021}\end{array}$ & $\begin{array}{l}+0.024 \\
-0.025\end{array}$ \\
\hline 185 & 0.255 & $\begin{array}{l}+0.023 \\
-0.019\end{array}$ & $\begin{array}{l}+0.022 \\
-0.023\end{array}$ \\
\hline 190 & 0.231 & $\begin{array}{l}+0.021 \\
-0.017\end{array}$ & $\begin{array}{l}+0.020 \\
-0.021\end{array}$ \\
\hline 195 & 0.210 & $\begin{array}{l}+0.019 \\
-0.015\end{array}$ & $\begin{array}{l}+0.019 \\
-0.020\end{array}$ \\
\hline 200 & 0.192 & $\begin{array}{l}+0.017 \\
-0.014\end{array}$ & $\begin{array}{l}+0.018 \\
-0.019\end{array}$ \\
\hline
\end{tabular}

Table 2

Cross sections (in pb) at the LHC $\left(\mu_{\mathrm{F}}=\mu_{R}=m_{H}\right)$ with $\sqrt{s}=10 \mathrm{TeV}$ using the MSTW2008 [15] parton densities.

\begin{tabular}{cccc|cccc|cccc}
\hline$m_{H}$ & $\sigma^{\text {best }}$ & Scale & PDF & $m_{H}$ & $\sigma^{\text {best }}$ & Scale & PDF & $m_{H}$ & $\sigma^{\text {best }}$ & Scale & PDF \\
\hline 100 & 44.12 & ${ }_{-4.44}^{+4.24}$ & ${ }_{-1.39}^{+1.07}$ & 170 & 15.63 & ${ }_{-1.30}^{+1.22}$ & ${ }_{-0.48}^{+0.39}$ & 240 & 7.81 & ${ }_{-0.58}^{+0.53}$ & ${ }_{-0.26}^{+0.23}$ \\
110 & 36.99 & ${ }_{-3.43}^{+3.43}$ & ${ }_{-1.14}^{+0.88}$ & 180 & 13.78 & ${ }_{-1.12}^{+1.05}$ & ${ }_{-0.42}^{+0.35}$ & 250 & 7.29 & ${ }_{-0.53}^{+0.49}$ & ${ }_{-0.25}^{+0.22}$ \\
120 & 31.48 & ${ }_{-2.83}^{+2.83}$ & ${ }_{-0.96}^{+0.75}$ & 190 & 12.20 & ${ }_{-0.97}^{+0.91}$ & ${ }_{-0.38}^{+0.32}$ & 260 & 6.83 & ${ }_{-0.49}^{+0.45}$ & ${ }_{-0.24}^{+0.21}$ \\
130 & 27.11 & ${ }_{-2.35}^{+2.48}$ & ${ }_{-0.82}^{+0.64}$ & 200 & 10.97 & ${ }_{-0.86}^{+0.80}$ & ${ }_{-0.35}^{+0.29}$ & 270 & 6.44 & ${ }_{-0.46}^{+0.42}$ & ${ }_{-0.23}^{+0.21}$ \\
140 & 23.58 & ${ }_{-2.10}^{+1.98}$ & ${ }_{-0.71}^{+0.56}$ & 210 & 9.98 & ${ }_{-0.77}^{+0.72}$ & ${ }_{-0.32}^{+0.27}$ & 280 & 6.11 & ${ }_{-0.43}^{+0.40}$ & ${ }_{-0.22}^{+0.20}$ \\
150 & 20.69 & ${ }_{-1.89}^{+1.69}$ & ${ }_{-0.62}^{+0.50}$ & 220 & 9.14 & ${ }_{-0.69}^{+0.64}$ & ${ }_{-0.30}^{+0.26}$ & 290 & 5.83 & ${ }_{-0.40}^{+0.37}$ & ${ }_{-0.22}^{+0.20}$ \\
160 & 18.07 & ${ }_{-1.53}^{+1.44}$ & ${ }_{-0.55}^{+0.44}$ & 230 & 8.42 & ${ }_{-0.63}^{+0.58}$ & ${ }_{-0.28}^{+0.24}$ & 300 & 5.61 & ${ }_{-0.38}^{+0.37}$ & ${ }_{-0.21}^{+0.19}$ \\
\hline
\end{tabular}

Table 3

Cross sections (in pb) at the LHC $\left(\mu_{F}=\mu_{R}=m_{H}\right)$ with $\sqrt{s}=14 \mathrm{TeV}$ using the MSTW2008 [15] parton densities.

\begin{tabular}{|c|c|c|c|c|c|c|c|c|c|c|c|}
\hline$m_{H}$ & $\sigma^{\text {best }}$ & Scale & PDF & $m_{H}$ & $\sigma^{\text {best }}$ & Scale & PDF & $m_{H}$ & $\sigma^{\text {best }}$ & Scale & PDF \\
\hline 100 & 74.58 & $\begin{array}{l}+7.18 \\
-7.54\end{array}$ & $\begin{array}{l}+1.86 \\
-2.45\end{array}$ & 170 & 28.46 & $\begin{array}{l}+2.22 \\
-2.39\end{array}$ & $\begin{array}{l}+0.65 \\
-0.84\end{array}$ & 240 & 15.10 & $\begin{array}{l}+1.03 \\
-1.12\end{array}$ & $\begin{array}{l}+0.37 \\
-0.45\end{array}$ \\
\hline 110 & 63.29 & $\begin{array}{r}+5.87 \\
-6.20\end{array}$ & $\begin{array}{r}+1.54 \\
-2.02\end{array}$ & 180 & 25.32 & $\begin{array}{r}+1.92 \\
-2.08\end{array}$ & $\begin{array}{l}+0.58 \\
-0.74\end{array}$ & 250 & 14.19 & $\begin{array}{r}+0.95 \\
-1.04\end{array}$ & $\begin{array}{l}+0.36 \\
-0.43\end{array}$ \\
\hline 120 & 54.48 & $\begin{array}{l}+4.88 \\
-5.18\end{array}$ & $\begin{array}{l}+1.30 \\
-1.70\end{array}$ & 190 & 22.63 & $\begin{array}{l}+1.68 \\
-1.83\end{array}$ & $\begin{array}{l}+0.52 \\
-0.66\end{array}$ & 260 & 13.41 & & $\begin{array}{l}+0.35 \\
-0.41\end{array}$ \\
\hline 130 & 47.44 & $\begin{array}{l}+4.12 \\
-4.38\end{array}$ & $\begin{array}{l}+1.12 \\
-1.45\end{array}$ & 200 & 20.52 & $\begin{array}{l}+1.49 \\
-1.63\end{array}$ & $\begin{array}{l}+0.48 \\
-0.60\end{array}$ & 270 & 12.74 & & $\begin{array}{l}+0.33 \\
-0.39\end{array}$ \\
\hline 140 & 41.70 & $\begin{array}{r}+3.47 \\
-3.75\end{array}$ & $\begin{array}{l}+0.97 \\
-1.25\end{array}$ & 210 & 18.82 & $\begin{array}{l}+1.34 \\
-1.47\end{array}$ & $\begin{array}{l}+0.45 \\
-0.55\end{array}$ & 280 & 12.17 & $\begin{array}{l}+0.78 \\
-0.86\end{array}$ & $\begin{array}{l}+0.33 \\
-0.38\end{array}$ \\
\hline 150 & 36.95 & $\begin{array}{l}+3.02 \\
-3.24\end{array}$ & $\begin{array}{l}+0.85 \\
-1.10\end{array}$ & 220 & 17.38 & $\begin{array}{l}+1.22 \\
-1.33\end{array}$ & $\begin{array}{l}+0.42 \\
-0.51\end{array}$ & 290 & 11.71 & & $\begin{array}{l}+0.32 \\
-0.37\end{array}$ \\
\hline 160 & 32.59 & $\begin{array}{r}+2.60 \\
-2.79\end{array}$ & $\begin{array}{r}+0.73 \\
-0.97\end{array}$ & 230 & 16.15 & $\begin{array}{r}+1.11 \\
-1.22\end{array}$ & $\begin{array}{r}+0.39 \\
-0.48\end{array}$ & 300 & 11.34 & $\begin{array}{l}+0.71 \\
-0.78\end{array}$ & $\begin{array}{l}+0.32 \\
-0.36\end{array}$ \\
\hline
\end{tabular}

tribution with respect to the previous analysis [7] results in an increase of the cross section from about $7 \%\left(m_{H}=115 \mathrm{GeV}\right)$ to $4 \%$ $\left(m_{H}=200 \mathrm{GeV}\right)$ at the Tevatron and from $9 \%\left(m_{H}=110 \mathrm{GeV}\right)$ to $2 \%\left(m_{H}=300 \mathrm{GeV}\right)$ at the LHC. The inclusion of the EW corrections results in an increase of the cross section by about $5 \%$ for $m_{H} \lesssim 160 \mathrm{GeV}$, and a decrease by about $2 \%$ for $200 \mathrm{GeV} \lesssim m_{H} \lesssim$ $300 \mathrm{GeV}$.

Our results for the Tevatron can be compared to those recently presented in Ref. [18], obtained using the same set of PDFs. Besides the different choice for the bottom-quark mass and the implementation of an effective Lagrangian calculation for the EW corrections, 
the main difference with our work arises in the calculation of the top-quark contribution to the cross section. In Ref. [18] the latter contribution is computed up to NNLO but choosing $\mu_{F}=\mu_{R}=$ $m_{H} / 2$, as an attempt to mimic the effects of soft-gluon resummation beyond NNLO. The final numerical differences at the Tevatron turn out to be small and of the order of a few per mille at the lowest masses, increasing to $2.5 \%$ at $m_{H}=200 \mathrm{GeV}$.

We now discuss the various sources of uncertainty affecting the cross sections presented in Tables 1,2 and 3 . The uncertainty basically has two origins: the one coming from the partonic cross sections, and the one arising from our limited knowledge of the PDFs.

Uncalculated higher-order QCD radiative corrections are the most important source of uncertainty on the partonic cross section. A method, which is customarily used in perturbative QCD calculations, to estimate their size is to vary the renormalization and factorization scales around the hard scale $m_{H}$. In general, this procedure can only give a lower limit on the true uncertainty. The uncertainty is quantified here as in Ref. [7]: we vary independently $\mu_{F}$ and $\mu_{R}$ in the range $0.5 m_{H} \leqslant \mu_{F}, \mu_{R} \leqslant 2 m_{H}$, with the constraint $0.5 \leqslant \mu_{F} / \mu_{R} \leqslant 2$. The results are reported in Tables 1,2 and 3 . The scale uncertainty is about $\pm 9-10 \%$ at the Tevatron and ranges from about $\pm 10 \%\left(m_{H}=110 \mathrm{GeV}\right)$ to about $\pm 7 \%\left(m_{H}=300 \mathrm{GeV}\right)$ at the LHC $(\sqrt{s}=10$ and $14 \mathrm{TeV})$. These results are consistent with those of Ref. [7]; in particular, we note that the effect of scale variations in our resummed calculation is considerably reduced with respect to the corresponding NNLO result. At NNLO the scale uncertainty is about $\pm 14 \%$ at the Tevatron and ranges from about $\pm 12 \%\left(m_{H}=110 \mathrm{GeV}\right)$ to about $\pm 9 \%$ $\left(m_{H}=300 \mathrm{GeV}\right)$ at the $\operatorname{LHC}(\sqrt{s}=10$ and $14 \mathrm{TeV})$. The reduction is more sizeable at the Tevatron, where the resummation effect is more important.

Another source of perturbative uncertainty on the partonic cross sections comes from the implementation of the EW corrections. Our results are obtained in the complete factorization scheme discussed above. The partial factorization scheme would lead to a change of our results ranging from about $-3 \%\left(m_{H}=\right.$ $115 \mathrm{GeV})$ to $+2 \%\left(m_{H}=200 \mathrm{GeV}\right)$ at the Tevatron and from about $-3 \%\left(m_{H}=110 \mathrm{GeV}\right)$ to $+1 \%\left(m_{H}=300 \mathrm{GeV}\right)$ at the LHC.

A different source of perturbative uncertainty comes from the use of the large- $m_{t}$ approximation in the computation of the partonic cross section beyond LO. The comparison between the exact NLO cross section and the one obtained in the large- $m_{t}$ approximation (but rescaled with the full Born result, including its exact dependence on $m_{t}$ ) shows that the approximation works well also for $m_{H}>m_{t}$. This is not accidental: the higher-order contributions to the cross section are dominated by relatively soft radiation, which is weakly sensitive to the mass of the heavy quark in the loop at Born level. This feature persists at NNLO and thus it is natural to assume that having normalized our resummed result with the exact $m_{t}$-dependent cross section, the uncertainty due to the large- $m_{t}$ approximation should be of the order of few percent, as it is at NLO. The effect of finite- $m_{t}$ corrections is discussed in Refs. [19,20].

The other important source of uncertainty in the cross section is the one coming from PDFs. Modern PDF sets let the user estimate the experimental uncertainty originating from the accuracy of the data points used to perform the fit. The most recent MSTW2008 NNLO set provides 40 different grids that allow us to evaluate the experimental uncertainties according to the procedure discussed in Ref. [11]. The outcoming uncertainties (at 90\% CL) are reported in Tables 1,2 and 3. At the Tevatron the effect ranges from $\pm 6 \%\left(m_{H}=115 \mathrm{GeV}\right)$ to about $\pm 10 \%\left(m_{H}=200 \mathrm{GeV}\right)$, while at the LHC it is about $\pm 3 \%( \pm 3-4 \%$ at $\sqrt{s}=10 \mathrm{TeV})$ in the mass range we have considered. We note that at the LHC the PDF uncertainty is quite small, and, in particular, it is sub- stantially smaller than the uncertainty from missing higher-order perturbative contributions, as estimated from scale variations. On the contrary, the PDF uncertainty at the Tevatron is larger. This is a consequence of the fact that the Tevatron probes relatively larger values of $x$, where the gluon density is less constrained. By using the MRST2006 set [14] our result for $m_{H}=170 \mathrm{GeV}$ at the Tevatron would be $\sigma^{\text {best (2006) }}=0.395_{-0.031}^{+0.036}$ (scale) ${ }_{-0.015}^{+0.017}(\mathrm{PDF})$, which is $13 \%$ smaller than the one reported in Table $1, \sigma^{\text {best }}=$ $0.349_{-0.027}^{+0.032}$ (scale) ${ }_{-0.029}^{+0.028}$ (PDF), and marginally compatible with it.

We finally point out that a related and important uncertainty is the one coming from the value of the QCD coupling. In modern PDF sets $\alpha_{S}\left(m_{Z}\right)$ is obtained together with the parton densities through a global fit to the available data, and thus there will be a correlation between the error on $\alpha_{S}\left(m_{Z}\right)$ and that on the gluon density. Since the gluon fusion process starts at $\mathcal{O}\left(\alpha_{\mathrm{S}}^{2}\right)$, it is easy to foresee that the uncertainty on $\alpha_{S}\left(m_{Z}\right)$ may have an important impact on the cross section. Neglecting correlations with the gluon density, a $3 \%$ uncertainty on $\alpha_{S}\left(m_{Z}\right)$ would lead to an effect of about $\pm 9-10 \%$ on the production cross section at both the Tevatron and the LHC.

To summarize, we have presented updated predictions for the cross section for Higgs boson production at the Tevatron and the LHC. The results are based on the most advanced theoretical information available at present for this observable, including softgluon resummation up to NNLL accuracy and two-loop EW corrections. In comparison with the central predictions of Ref. [7], at the Tevatron the difference ranges from $+9 \%$ to $-9 \%$ for $115 \mathrm{GeV} \lesssim$ $m_{H} \lesssim 200 \mathrm{GeV}$. At the LHC the effect goes from $+30 \%$ to $+9 \%$ for $115 \mathrm{GeV} \lesssim m_{H} \lesssim 300 \mathrm{GeV}$, and is mostly due to the new PDFs. We have then reviewed [7] the uncertainties that affect the Higgs production cross section, and we have shown that they are still relatively large, especially at the Tevatron. The above uncertainties should be taken into account in Higgs boson searches and studies at both the Tevatron and the LHC.

\section{Acknowledgements}

We thank Babis Anastasiou and Christian Sturm for useful discussions. We are grateful to Stefano Catani for helpful discussions and comments on the manuscript. The work of D.deF. was partially supported by ANPCYT, UBA-CyT and CONICET. D.deF. is grateful to the US Department of Energy (Contract No. DE-AC02-98CH10886) for providing the facilities essential for the completion of his work.

\section{References}

[1] R. Barate, et al., LEP Working Group for Higgs boson searches, ALEPH Collaboration, DELPHI Collaboration, L3 Collaboration, OPAL Collaboration, Phys. Lett. B 565 (2003) 61.

[2] J. Qian, CDF Collaboration, DØ Collaboration, arXiv:0812.3979 [hep-ex], Proc. Phys. LHC, Split, Croatia, September 2008, in press.

[3] S. Dawson, Nucl. Phys. B 359 (1991) 283.

[4] A. Djouadi, M. Spira, P.M. Zerwas, Phys. Lett. B 264 (1991) 440; M. Spira, A. Djouadi, D. Graudenz, P.M. Zerwas, Nucl. Phys. B 453 (1995) 17.

[5] R.V. Harlander, W.B. Kilgore, Phys. Rev. Lett. 88 (2002) 201801; C. Anastasiou, K. Melnikov, Nucl. Phys. B 646 (2002) 220; V. Ravindran, J. Smith, W.L. van Neerven, Nucl. Phys. B 665 (2003) 325.

[6] M. Kramer, E. Laenen, M. Spira, Nucl. Phys. B 511 (1998) 523; S. Catani, D. de Florian, M. Grazzini, JHEP 0105 (2001) 025; R.V. Harlander, W.B. Kilgore, Phys. Rev. D 64 (2001) 013015.

[7] S. Catani, D. de Florian, M. Grazzini, P. Nason, JHEP 0307 (2003) 028.

[8] S. Moch, A. Vogt, Phys. Lett. B 631 (2005) 48.

[9] E. Laenen, L. Magnea, Phys. Lett. B 632 (2006) 270.

[10] A. Idilbi, X.d. Ji, J.P. Ma, F. Yuan, Phys. Rev. D 73 (2006) 077501.

[11] A.D. Martin, R.G. Roberts, W.J. Stirling, R.S. Thorne, Eur. Phys. J. C 28 (2003) 455.

[12] S. Moch, J.A.M. Vermaseren, A. Vogt, Nucl. Phys. B 688 (2004) 101; A. Vogt, S. Moch, J.A.M. Vermaseren, Nucl. Phys. B 691 (2004) 129.

[13] W.L. van Neerven, A. Vogt, Phys. Lett. B 490 (2000) 111.

[14] A.D. Martin, W.J. Stirling, R.S. Thorne, G. Watt, Phys. Lett. B 652 (2007) 292. 
[15] A.D. Martin, W.J. Stirling, R.S. Thorne, G. Watt, arXiv:0901.0002 [hep-ph].

[16] U. Aglietti, R. Bonciani, G. Degrassi, A. Vicini, Phys. Lett. B 595 (2004) 432; G. Degrassi, F. Maltoni, Phys. Lett. B 600 (2004) 255;

U. Aglietti, R. Bonciani, G. Degrassi, A. Vicini, Contributed to the TeV4LHC Workshop, Brookhaven, Upton, New York, February 2005, arXiv:hep-ph/ 0610033.

[17] S. Actis, G. Passarino, C. Sturm, S. Uccirati, Phys. Lett. B 670 (2008) 12;
S. Actis, G. Passarino, C. Sturm, S. Uccirati, Nucl. Phys. B 811 (2009) 182.

[18] C. Anastasiou, R. Boughezal, F. Petriello, arXiv:0811.3458v2 [hep-ph].

[19] M. Schreck, M. Steinhauser, Phys. Lett. B 655 (2007) 148.

20] S. Marzani, R.D. Ball, V. Del Duca, S. Forte, A. Vicini, Nucl. Phys. B 800 (2008) 127;

S. Marzani, R.D. Ball, V. Del Duca, S. Forte, A. Vicini, Nucl. Phys. B (Proc Suppl.) 186 (2009) 98. 